\title{
Dynamic Surface Control and Its Application to Lateral Vehicle Control
}

\author{
Bongsob Song, ${ }^{1}$ J. Karl Hedrick, ${ }^{2}$ and Yeonsik Kang ${ }^{3}$ \\ ${ }^{1}$ Department of Mechanical Engineering, Ajou University, Suwon 443-749, Republic of Korea \\ ${ }^{2}$ Department of Mechanical Engineering, University of California, Berkeley, CA 94720, USA \\ ${ }^{3}$ Department of Automotive Engineering, Kookmin University, Seoul 136-702, Republic of Korea
}

Correspondence should be addressed to Bongsob Song; bsong@ajou.ac.kr

Received 4 December 2013; Accepted 31 March 2014; Published 29 April 2014

Academic Editor: Ilse Cervantes Camacho

Copyright (C) 2014 Bongsob Song et al. This is an open access article distributed under the Creative Commons Attribution License, which permits unrestricted use, distribution, and reproduction in any medium, provided the original work is properly cited.

This paper extends the design and analysis methodology of dynamic surface control (DSC) in Song and Hedrick, 2011, for a more general class of nonlinear systems. When rotational mechanical systems such as lateral vehicle control and robot control are considered for applications, sinusoidal functions are easily included in the equation of motions. If such a sinusoidal function is used as a forcing term for DSC, the stability analysis faces the difficulty due to highly nonlinear functions resulting from the low-pass filter dynamics. With modification of input variables to the filter dynamics, the burden of mathematical analysis can be reduced and stability conditions in linear matrix inequality form to guarantee the quadratic stability via DSC are derived for the given class of nonlinear systems. Finally, the proposed design and analysis approach are applied to lateral vehicle control for forward automated driving and backward parallel parking at a low speed as well as an illustrative example.

\section{Introduction}

The dynamic surface control (DSC), one of robust nonlinear control techniques, has been developed with a wide spectrum of applications including throttle/brake control on automated vehicles [1], underactuated ship control [2], and robot control [3]. This control technique is a dynamic extension of multiple sliding surface control with a series of first order low-pass filters to avoid an "explosion of terms" [4]. The existence of DSC gains and filter time constants for semiglobal stability was theoretically proved in [4]. Recently, a noble analysis method in the framework of convex optimization has been introduced to allow us to find a quadratic Lyapunov function numerically for a class of nonlinear systems called "strictfeedback" form as follows [3]:

$$
\begin{aligned}
& \dot{x}_{i}=x_{i+1}+f_{1}\left(x_{1}, \ldots, x_{i}\right) \quad \text { for } i=1, \ldots, n-1, \\
& \dot{x}_{n}=u+f_{n}\left(x_{1}, \ldots, x_{n}\right) .
\end{aligned}
$$

Furthermore, if $x_{i+1}$ in (1) is replaced by $g_{i+1}\left(x_{i+1}\right)$ where $g_{i+1}$ and $\left[\partial g_{i+1} / \partial x\right]$ are continuous and invertible, the design procedure proposed by Swaroop et al. [4] and Gerdes and Hedrick [5] can be still applied for the given system.

However, this replacement induces another highly nonlinear function resulting from the low-pass filter error dynamics when stability analysis is performed. The following example illustrates the design approach of DSC as well as the difficulty that this paper seeks to solve:

$$
\begin{aligned}
& \dot{x}_{1}=\tan x_{2}+f_{1}\left(x_{1}\right), \\
& \dot{x}_{2}=u,
\end{aligned}
$$

where $f_{1}$ and $\left[\partial f_{1} / \partial x_{1}\right]$ are continuous on $\mathscr{D}$; for example, $\mathscr{D}=\left\{x \in \mathfrak{R}^{2}|| x_{1}|\leq 1,| x_{2} \mid \leq \pi / 4\right\}$; thus both $\tan x_{2}$ and $f_{1}$ are bounded on $\mathscr{D}$. The control objective is to stabilize the system; that is, $x_{1} \rightarrow 0$. First, define the first error surface as $S_{1}=x_{1}$. After taking its derivative along the trajectory of (2)

$$
\dot{S}_{1}=\tan x_{2}+f_{1} .
$$

Then, the synthetic input, which is forced to drive $S_{1} \rightarrow 0$, is derived as

$$
\tan \bar{x}_{2}=-f_{1}-K S_{1} \Longrightarrow \bar{x}_{2}=\tan ^{-1}\left(-f_{1}-K S_{1}\right),
$$


where $K$ is a controller gain. We now define the second sliding surface $S_{2}=x_{2}-x_{2 d}$, where $x_{2 d}$ equals $\bar{x}_{2}$ passed through a first order low-pass filter; that is,

$$
\tau \dot{x}_{2 d}+x_{2 d}=\bar{x}_{2}, \quad x_{2 d}(0)=\bar{x}_{2}(0)
$$

where $\tau$ is the filter time constant. Finally, the control input is derived as

$$
u=\dot{x}_{2 d}-K S_{2}=\frac{\bar{x}_{2}-x_{2 d}}{\tau}-K S_{2} .
$$

Next, the stability analysis is investigated based on the closed-loop dynamics as suggested in [3]. If both $\tan \bar{x}_{2}$ and $\tan x_{2 d}$ are added and subtracted in (2) and $u$ in (7) is put in (3), the closed-loop dynamics is written as

$$
\begin{aligned}
& \dot{x}_{1}=\left(\tan x_{2}-\tan x_{2 d}\right)+\left(\tan x_{2 d}-\tan \bar{x}_{2}\right)+\tan \bar{x}_{2}+f_{1}, \\
& \dot{x}_{2}=\dot{x}_{2 d}-K S_{2} .
\end{aligned}
$$

By use of (5) and definitions of $S_{1}$ and $S_{2},(8)$ is rewritten as

$$
\begin{aligned}
& \dot{S}_{1}=\left(\tan x_{2}-\tan x_{2 d}\right)+\left(\tan x_{2 d}-\tan \bar{x}_{2}\right)-K S_{1}, \\
& \dot{S}_{2}=-K S_{2} .
\end{aligned}
$$

Since the first order low-pass filter in (6) is added, the filter dynamics should be included in the closed-loop dynamics for stability analysis. After defining the filter error, $\xi=x_{2 d}-\bar{x}_{2}$, the augmented closed-loop dynamics is summarized as

$$
\begin{aligned}
\dot{S}_{1} & =-K S_{1}+\left(\tan x_{2}-\tan x_{2 d}\right)+\left(\tan x_{2 d}-\tan \bar{x}_{2}\right) \\
& :=-K S_{1}+w_{1}+w_{2}, \\
\dot{S}_{2} & =-K S_{2}, \\
\dot{\xi} & =-\frac{\xi}{\tau}-\frac{d}{d t}\left\{\tan ^{-1}\left(-f_{1}-K S_{1}\right)\right\} \\
& =-\frac{\xi}{\tau}+\frac{1}{1+\left(f_{1}+K S_{1}\right)^{2}}\left(\dot{f}_{1}+K \dot{S}_{1}\right) \\
& :=-\frac{\xi}{\tau}+\eta\left(S_{1}\right)\left(\dot{f}_{1}+K \dot{S}_{1}\right) .
\end{aligned}
$$

Since the function, $\tan x$, is locally Lipschitz, there exists $\gamma>$ 0 such that

$$
\begin{aligned}
\left|\tan x_{2}-\tan x_{2 d}\right| & \leq \gamma\left|x_{2}-x_{2 d}\right|=\gamma\left|S_{2}\right|,\left|\tan x_{2 d}-\tan \bar{x}_{2}\right| \\
& \leq \gamma\left|x_{2 d}-\bar{x}_{2}\right|=\gamma|\xi|
\end{aligned}
$$

where $\gamma$ is a Lipschitz constant on $\mathscr{D}$. Using the continuity of $f_{1}$ and $\left[\partial f_{1} / \partial x_{1}\right]$ in (2), it is also shown that the last term of the third row in (10) is bounded on $\mathscr{D}$. Therefore, the existence of the controller gain $K$ and filter time constant $\tau$ for semiglobal stability can be shown as suggested in [5].

However, this fact does not tell us whether the closedloop system is stable for the given $K$ and $\tau$. To answer the question, we may need to find a Lyapunov function candidate explicitly and one of the possible analysis approaches is based on linear matrix inequality. To apply this approach to (10), it is necessary to write it in matrix form as

$$
\begin{aligned}
& {\left[\begin{array}{ccc}
1 & 0 & 0 \\
0 & 1 & 0 \\
-K \eta & 0 & 1
\end{array}\right]\left[\begin{array}{c}
\dot{S}_{1} \\
\dot{S}_{2} \\
\dot{\xi}
\end{array}\right]} \\
& \quad=\left[\begin{array}{ccc}
-K & 0 & 0 \\
0 & -K & 0 \\
0 & 0 & -\frac{1}{\tau}
\end{array}\right]\left[\begin{array}{l}
S_{1} \\
S_{2} \\
\xi
\end{array}\right]+\left[\begin{array}{lll}
1 & 1 & 0 \\
0 & 0 & 0 \\
0 & 0 & \eta
\end{array}\right]\left[\begin{array}{c}
w_{1} \\
w_{2} \\
\dot{f}
\end{array}\right] .
\end{aligned}
$$

While the next procedure is to investigate whether (12) is in a class of linear differential inclusions classified in [6], the inclusion of the nonlinear function $\eta\left(S_{1}\right)$ in (12) results in the mathematical difficulty of stability analysis.

The contribution of this paper is to extend a design and analysis methodology of DSC for a more general class of nonlinear systems as shown in (2) and (3). The consideration of this class of nonlinear systems is motivated when rotational mechanical systems are considered for applications; that is, sinusoidal functions are in general included in the equation of motions. As one of the applications, the proposed control approach is applied to lateral vehicle control for forward automated driving and backward parallel parking at a low speed. Finally, its performance will be validated via simulations.

\section{Problem Statement}

Consider the class of nonlinear systems

$$
\begin{aligned}
\dot{x}_{1} & =g_{2}\left(x_{2}\right)+f_{1}\left(x_{1}\right), \\
\dot{x}_{2} & =g_{3}\left(x_{3}\right)+f_{2}\left(x_{1}, x_{2}\right), \\
& \vdots \\
\dot{x}_{n-1} & =g_{n}\left(x_{n}\right)+f_{n-1}\left(x_{1}, \ldots, x_{n-1}\right), \\
\dot{x}_{n} & =u+f_{n}\left(x_{1}, \ldots, x_{n}\right),
\end{aligned}
$$

where $f_{i}$ and $\left[\partial f_{i} / \partial x\right]$ are continuous on $\mathscr{D}_{i} \subset \mathscr{D} \subset \mathfrak{R}^{n}$ and $f_{i}: \mathscr{D}_{i} \rightarrow \mathfrak{R}$ is in strict-feedback form in the sense that the $f_{i}$ depends only on $x_{1}, \ldots, x_{i}$. It is implied that $f_{i}$ is locally Lipschitz and $\left[\partial f_{i}(x) / \partial x\right]$ is bounded on $\mathscr{D}_{i}$ [7]. Therefore, there exists a constant $\gamma_{i}>0$ such that

$$
\left\|\frac{\partial f_{i}}{\partial x}\right\|=\left\|\left[\begin{array}{lll}
\frac{\partial f_{i}}{\partial x_{1}} & \cdots & \frac{\partial f_{i}}{\partial x_{i}}
\end{array}\right]\right\|:=\left\|J_{i}\right\| \leq \gamma_{i}
$$

for all $x$ on $\mathscr{D}_{i}$.

The nonlinear function $g_{i}$ is also locally Lipschitz; that is, there exists a constant $\lambda_{i}>0$ such that

$$
\left|g_{i}(a)-g_{i}(b)\right|=\lambda_{i}|a-b|, \quad i=2, \ldots, n .
$$

In addition, there exist differentiable functions $q_{i}: \mathscr{E}_{i} \rightarrow \mathfrak{R}$, where $\mathscr{E}_{i}=\left\{y \in \mathfrak{R} \mid y=g_{i}(x)\right.$ for all $\left.x \in \mathscr{D}_{i}\right\}$, which are inverses of the $g_{i}$ in the sense that

$$
q_{i}\left(g_{i}(c)\right)=c, \quad i=2, \ldots, n,
$$


and $\left[\partial q_{i} / \partial g_{i}\right]$ is bounded on $\mathscr{D}$; that is, there exists a constant $\delta>0$ such that

$$
\left\|\left[\begin{array}{lll}
\frac{\partial q_{2}}{\partial g_{2}} & \cdots & \frac{\partial q_{n}}{\partial g_{n}}
\end{array}\right]\right\| \leq \delta .
$$

\section{Analysis and Design of DSC}

3.1. Design Procedure. Although the proposed design procedure is quite similar to the standard one described in [4], an outline of the design procedure is as follows. Define the first error surface as $S_{1}:=x_{1}-x_{1 d}$, where $x_{1 d}$ is the desired value as the control objective. After taking the time derivative of $S_{1}$ along the trajectory of (13),

$$
\dot{S}_{1}=g_{2}\left(x_{2}\right)+f_{1}\left(x_{1}\right)-\dot{x}_{1 d} .
$$

The surface error $S_{1}$ will converge to zero if $S_{1} \dot{S}_{1}<0$; however there is no direct control over the surface dynamics. If $g_{2}$ is considered as the forcing term for the surface dynamics, then the sliding condition outside some boundary layer is satisfied if $g_{2}=\bar{g}_{2}$, where

$$
\bar{g}_{2}\left(\bar{x}_{2}\right)=\dot{x}_{1 d}-f_{1}\left(x_{1}\right)-K_{1} S_{1}, \quad \bar{x}_{2}=q_{2}\left(\bar{g}_{2}\right),
$$

where $q_{2}$ is the inverse of $g_{2}$.

The next step is to force $x_{2} \rightarrow \bar{x}_{2}$, so define $S_{2}:=x_{2}-x_{2 d}$, where $x_{2 d}=q_{2}\left(g_{2 d}\right)$ and $g_{2 d}$ is obtained after passing through a first order low-pass filter; that is,

$$
\tau_{2} \dot{g}_{2 d}+g_{2 d}=\bar{g}_{2}, \quad g_{2 d}(0):=\bar{g}_{2}(0) .
$$

It is noted that this procedure is different from the one explained in the introduction. That is, $\bar{g}_{2}$ instead of $\bar{x}_{2}$ passes through the filter and the inverse function of the filtered signal is used to define $x_{2 d}$. After taking a derivative of $S_{2}$ along the trajectory of (13), the resulting synthesis term, $\bar{g}_{3}$, is derived as

$$
\bar{g}_{3}\left(\bar{x}_{3}\right)=\dot{x}_{2 d}-f_{2}\left(x_{1}, x_{2}\right)-K_{2} S_{2}, \quad \bar{x}_{3}=q_{3}\left(\bar{g}_{3}\right),
$$

where

$$
\dot{x}_{2 d}=\frac{\partial q_{2}}{\partial g_{2 d}} \dot{g}_{2 d}=\frac{\partial q_{2}}{\partial g_{2 d}} \frac{\bar{g}_{2}-g_{2 d}}{\tau_{2}}
$$

and the last equality comes from (20).

Similarly, continuing this process for each consecutive state, define the $i$ th error surface as $S_{i}=x_{i}-x_{i d}$ where $x_{i d}=q_{i}\left(g_{i d}\right)$ and $\bar{g}_{i+1}$ is

$$
\begin{aligned}
\bar{g}_{i+1}\left(\bar{x}_{i+1}\right) & =\dot{x}_{i d}-f_{i}\left(x_{1}, \ldots, x_{i}\right)-K_{i} S_{i}, \\
\bar{x}_{i+1} & =q_{i+1}\left(\bar{g}_{i+1}\right),
\end{aligned}
$$

where

$$
\dot{x}_{i d}=\frac{\partial q_{i}}{\partial g_{i d}} \dot{g}_{i d}=\frac{\partial q_{i}}{\partial g_{i d}} \frac{\bar{g}_{i}-g_{i d}}{\tau_{i}} .
$$

Then, $g_{(i+1) d}$ is obtained by filtering $\bar{g}_{i+1}$; that is,

$$
\tau_{i+1} \dot{g}_{(i+1) d}+g_{(i+1) d}=\bar{g}_{i+1}, \quad g_{(i+1) d}(0):=\bar{g}_{i+1}(0) \text {. }
$$

After continuing this procedure for $1 \leq i \leq n-1$, define $S_{n}:=x_{n}-x_{n d}$, where $x_{n d}=q_{n}\left(g_{n d}\right)$. Finally, the control input is derived as

$$
u=\dot{x}_{n d}-f_{n}\left(x_{1}, \ldots, x_{n}\right)-K_{n} S_{n},
$$

where

$$
\dot{x}_{n d}=\frac{\partial q_{n}}{\partial g_{n d}} \dot{g}_{n d}=\frac{\partial q_{n}}{\partial g_{n d}} \frac{\bar{g}_{n}-g_{n d}}{\tau_{n}} .
$$

3.2. Augmented Error Dynamics. The closed-loop error dynamics will be derived for stability analysis in this section. After subtracting and adding $\bar{g}_{i+1}$ and $g_{(i+1) d}$ and using (26) in $u$, the closed-loop dynamics of (13) can be written as

$$
\begin{array}{r}
\dot{x}_{i}=\bar{g}_{i+1}+\left[g_{i+1}-g_{(i+1) d}\right]+\left[g_{(i+1) d}-\bar{g}_{i+1}\right]+f_{i} \\
\text { for } i=1, \ldots, n-1,
\end{array}
$$

$$
\dot{x}_{n}=\dot{x}_{n d}-K_{n} S_{n}
$$

By use of (23) and the definition of error surfaces, the above equations can be described in terms of the error surfaces of DSC as follows:

$$
\begin{aligned}
& \dot{S}_{i}=-K_{i} S_{i}+h_{i+1}+\left[g_{(i+1) d}-\bar{g}_{i+1}\right] \text { for } i=1, \ldots, n-1, \\
& \dot{S}_{n}=-K_{n} S_{n},
\end{aligned}
$$

where $h_{i+1}=g_{i+1}-g_{(i+1) d}$.

In addition, we need to consider the augmented error dynamics due to inclusion of a set of the first order low-pass filters. Let us define the filter error as $\xi_{i}:=g_{i d}-\bar{g}_{i}$ for $2 \leq i \leq$ $n$. Then, the filter dynamics are

$$
\dot{\xi}_{i}=\dot{g}_{i d}-\frac{d \bar{g}_{i}}{d t}=-\frac{\xi_{i}}{\tau_{i}}-\frac{d \bar{g}_{i}}{d t},
$$

where the last equality comes from (25). By taking a derivative of (23), we can write $d \bar{g}_{i} / d t$ as

$$
\begin{aligned}
& \frac{d \bar{g}_{2}}{d t}=-\dot{f}_{1}+\ddot{x}_{1 d}-K_{1} \dot{S}_{1}, \\
& \frac{d \bar{g}_{i}}{d t}=-\dot{f}_{i-1}+\ddot{x}_{(i-1) d}-K_{i-1} \dot{S}_{i-1} \quad \text { for } i=3, \ldots, n .
\end{aligned}
$$

Combining (30) with (31), we have the filter error dynamics,

$$
\begin{aligned}
\dot{\xi}_{2}-K_{1} \dot{S}_{1} & =-\frac{\xi_{2}}{\tau_{2}}+\dot{f}_{1}-\ddot{x}_{1 d}, \\
\dot{\xi}_{i}-K_{i-1} \dot{S}_{i-1} & =-\frac{\xi_{i}}{\tau_{i}}+\dot{f}_{i-1}-\ddot{x}_{(i-1) d} \quad \text { for } i=3, \ldots, n .
\end{aligned}
$$

Therefore, the overall error dynamics, (29) and (32), can be given as

$$
\begin{aligned}
\dot{S}_{i} & =-K_{i} S_{i}+\xi_{i+1}+h_{i+1} \quad \text { for } i=1, \ldots, n-1, \\
\dot{S}_{n} & =-K_{n} S_{n}, \\
\dot{\xi}_{j+1}-K_{j} \dot{S}_{j} & =-\frac{\xi_{j+1}}{\tau_{j+1}}+\dot{f}_{j}-\ddot{x}_{j d} \quad \text { for } j=1, \ldots, n-1 .
\end{aligned}
$$


Furthermore, (33) can be written in matrix form as follows:

$$
\begin{aligned}
{\left[\begin{array}{cc}
\mathbf{I}_{n} & \mathbf{0} \\
-\mathbf{K}_{0} & \mathbf{I}_{n-1}
\end{array}\right]\left[\begin{array}{l}
\dot{S} \\
\dot{\xi}
\end{array}\right] } \\
=\left[\begin{array}{cc}
-\mathbf{K} & \mathbf{I}_{n \times(n-1)} \\
\mathbf{0} & -\boldsymbol{\Gamma}
\end{array}\right]\left[\begin{array}{l}
S \\
\xi
\end{array}\right]+\left[\begin{array}{cc}
\mathbf{I}_{n \times(n-1)} & \mathbf{0} \\
\mathbf{0} & \mathbf{I}_{n-1}
\end{array}\right]\left[\begin{array}{c}
h \\
\dot{f}
\end{array}\right] \\
+\left[\begin{array}{c}
\mathbf{0} \\
-\mathbf{I}_{n-1}
\end{array}\right] \ddot{x}_{d}
\end{aligned}
$$

where the vectors are defined as

$$
\begin{aligned}
S & =\left[\begin{array}{lll}
S_{1} & \cdots & S_{n}
\end{array}\right]^{T} \in \mathfrak{R}^{n}, \quad \xi=\left[\begin{array}{lll}
\xi_{2} & \cdots & \xi_{n}
\end{array}\right]^{T} \in \mathfrak{R}^{n-1}, \\
h & =\left[\begin{array}{lll}
h_{2} & \cdots & h_{n}
\end{array}\right]^{T} \in \mathfrak{R}^{n-1}, \\
\dot{f} & =\left[\begin{array}{llll}
\dot{f}_{1} & \dot{f}_{2} & \cdots & \dot{f}_{n-1}
\end{array}\right]^{T} \in \mathfrak{R}^{n-1}, \\
\ddot{x}_{d} & =\left[\begin{array}{lll}
\ddot{x}_{1 d} & \cdots & \ddot{x}_{(n-1) d}
\end{array}\right]^{T} \in \mathfrak{R}^{n-1}
\end{aligned}
$$

and the submatrices are

$$
\begin{aligned}
\mathbf{K} & =\operatorname{diag}\left(K_{1}, \ldots, K_{n}\right), \\
\mathbf{K}_{0} & =\left[\operatorname{diag}\left(K_{1}, \ldots, K_{n-1}\right) 0_{n-1}\right] \in \mathfrak{R}^{(n-1) \times n}, \\
\Gamma & =\operatorname{diag}\left(\frac{1}{\tau_{2}}, \ldots, \frac{1}{\tau_{n}}\right) \in \mathfrak{R}^{(n-1) \times(n-1)} .
\end{aligned}
$$

Since the first block matrix in (34) is invertible such that

$$
\left[\begin{array}{cc}
\mathbf{I}_{n} & \mathbf{0} \\
-\mathbf{K}_{0} & \mathbf{I}_{n-1}
\end{array}\right]^{-1}=\left[\begin{array}{cc}
\mathbf{I}_{n} & \mathbf{0} \\
\mathbf{K}_{0} & \mathbf{I}_{n-1}
\end{array}\right],
$$

after multiplying the inverse matrix to both sides in (34), the augmented closed-loop error dynamics are rewritten as

$$
\begin{aligned}
\frac{d}{d t}\left[\begin{array}{l}
S \\
\xi
\end{array}\right]= & {\left[\begin{array}{cc}
-\mathbf{K} & \mathbf{I}_{n \times(n-1)} \\
\mathbf{K}_{0} \mathbf{K} & \Lambda
\end{array}\right]\left[\begin{array}{l}
S \\
\xi
\end{array}\right] } \\
& +\left[\begin{array}{cc}
\mathbf{I}_{n \times(n-1)} & \mathbf{0} \\
\mathbf{K}_{1:(n-1)} & \mathbf{I}_{n-1}
\end{array}\right]\left[\begin{array}{c}
h \\
m
\end{array}\right]+\left[\begin{array}{c}
\mathbf{0} \\
-\mathbf{I}_{n-1}
\end{array}\right] \ddot{x}_{d},
\end{aligned}
$$

where

$$
\mathbf{K}_{1:(n-1)}=\operatorname{diag}\left(K_{1}, \ldots, K_{n-1}\right), \quad \boldsymbol{\Lambda}=\mathbf{K}_{1:(n-1)}-\mathbf{\Gamma} .
$$
(29)

Since $\dot{x}_{j}$ is written in a function of $S, \xi, h$, and $\dot{x}_{1}$ based on

$$
\begin{aligned}
\dot{x}_{1}= & -K_{1} S_{1}+\xi_{2}+h_{2}+\dot{x}_{1 d}, \\
\dot{x}_{i}= & -K_{i} S_{i}+\xi_{i+1}+h_{i+1}+\dot{x}_{i d}=-K_{i} S_{i}+\xi_{i+1} \\
& +h_{i+1}-\frac{\partial q_{i}}{\partial g_{i d}} \frac{\xi_{i}}{\tau_{i}} \quad \text { for } i=2, \ldots, n-1, \\
\dot{x}_{n}= & -K_{n} S_{n}+\dot{x}_{n d}=-K_{n} S_{n}-\frac{\partial q_{n}}{\partial g_{n d}} \frac{\xi_{n}}{\tau_{n}},
\end{aligned}
$$

the time derivative of $f_{i}$ can be decomposed into three parts as follows:

$$
\begin{aligned}
\dot{f}_{i}= & \sum_{j=1}^{i} \frac{\partial f_{i}}{\partial x_{j}} \dot{x}_{j} \\
= & {\left[\begin{array}{lll}
\frac{\partial f_{i}}{\partial x_{1}} & \cdots & \frac{\partial f_{i}}{\partial x_{i}}
\end{array}\right] } \\
& \left.\times\left(\begin{array}{cccccccc}
-K_{1} & 0 & \cdots & 0 & 1 & 0 & \cdots & 0 \\
0 & -K_{2} & \cdots & 0 & 0 & 1 & \cdots & 0 \\
\vdots & \vdots & \ddots & \vdots & \vdots & \vdots & \ddots & \vdots \\
0 & 0 & \cdots & -K_{i} & 0 & 0 & \cdots & 1
\end{array}\right] z+\left[\begin{array}{c}
h_{2} \\
h_{3} \\
\vdots \\
h_{i+1}
\end{array}\right]\right) \\
& +\left[\begin{array}{ccccc}
0 \\
\frac{\partial f_{i}}{\partial x_{1}} \\
\cdots & \frac{\partial f_{i}}{\partial x_{i}}
\end{array}\right]\left[\begin{array}{c}
0 \\
-\frac{\partial q_{2}}{\partial g_{2 d}} \frac{\xi_{2}}{\tau_{2}} \\
\vdots \\
-\frac{\partial q_{i}}{\partial g_{i d}} \frac{\xi_{i}}{\tau_{i}}
\end{array}\right]+\frac{\partial f_{i}}{\partial x_{1}} \dot{x}_{1 d} \\
:= & p_{1 i}+p_{2 i}+p_{3 i} .
\end{aligned}
$$

Therefore, (38) is rewritten as

$$
\begin{aligned}
\frac{d}{d t}\left[\begin{array}{l}
S \\
\xi
\end{array}\right]= & {\left[\begin{array}{cc}
-\mathbf{K} & \mathbf{I}_{n \times(n-1)} \\
-\mathbf{K}_{0} \mathbf{K} & \Lambda
\end{array}\right]\left[\begin{array}{l}
S \\
\xi
\end{array}\right] } \\
& +\left[\begin{array}{ccc}
\mathbf{I}_{n \times(n-1)} & \mathbf{0} & \mathbf{0} \\
\mathbf{K}_{1:(n-1)} & \mathbf{I}_{n-1} & \mathbf{I}_{n-1}
\end{array}\right]\left[\begin{array}{c}
h \\
p_{1} \\
p_{2}
\end{array}\right] \\
& +\left[\begin{array}{cc}
\mathbf{0} & \mathbf{0} \\
\mathbf{I}_{n-1} & -\mathbf{I}_{n-1}
\end{array}\right]\left[\begin{array}{c}
p_{3} \\
\ddot{x}_{d}
\end{array}\right] \\
\Longrightarrow & \dot{z}=A_{c l} z+B_{w} w+B_{r} r
\end{aligned}
$$

where the error state $z=\left[\begin{array}{ll}S^{T} & \xi^{T}\end{array}\right]^{T} \in \mathfrak{R}^{2 n-1}:=\mathfrak{R}^{n_{z}}, w=$ $\left[\begin{array}{lll}h^{T} & p_{1}^{T} & p_{2}^{T}\end{array}\right]^{T} \in \mathfrak{R}^{3 n-3}:=\mathfrak{R}^{n_{w}}$, and $r=\left[\begin{array}{cc}p_{3}^{T} & \ddot{x}_{1 d}\end{array}\right]^{T} \in \mathfrak{R}^{n}$.

Finally, we need to determine the upper bound of $w$ in (42). Using the assumptions (15) in Section 2, the upper bound of $w_{i}$ for $i=1, \ldots, n-1$ is

$$
\begin{aligned}
\left|w_{i}\right|=\left|h_{i+1}\right| & =\left|g_{i+1}-g_{(i+1) d}\right| \leq \lambda_{i+1}\left|x_{i+1}-x_{(i+1) d}\right| \\
& =\lambda_{i+1}\left|S_{i+1}\right|:=\left|C_{z i} z\right| .
\end{aligned}
$$

Using (14), the upper bound of $w_{i}$ for $i=n, \ldots, 2 n-2$ is

$$
\begin{aligned}
\left|w_{i}\right| & =\left|p_{1 j}\right|=\left\|J_{j}\left(C_{z} z+D_{w} w\right)\right\| \leq \gamma_{j}\left\|\widetilde{C}_{z j} z+\widetilde{D}_{w j} w\right\| \\
& :=\left\|C_{z i} z+D_{w i} w\right\|, \quad j=1, \ldots, n-1,
\end{aligned}
$$


where $J_{j}=\left[\partial f_{j} / \partial x_{1} \cdots \partial f_{j} / \partial x_{j}\right], C_{z i}=\gamma_{j} \widetilde{C}_{z j}$, and $D_{w i}=$ $\gamma_{j} \widetilde{D}_{w j}$. Similarly, the upper bound of $w_{i}$ for $i=2 n-1, \ldots, n_{w}$ is obtained using (17)

$$
\begin{aligned}
\left|w_{i}\right| & =\left|p_{2 j}\right| \\
& =\left\|-\left[\frac{\partial f_{j}}{\partial x_{2}} \frac{\partial q_{2}}{\partial g_{2 d}} \cdots \frac{\partial f_{j}}{\partial x_{j}} \frac{\partial q_{j}}{\partial g_{j d}}\right]\left[\frac{\xi_{2}}{\tau_{2}} \ldots \frac{\xi_{i}}{\tau_{i}}\right]^{T}\right\| \\
& \leq \delta \gamma_{j}\left\|\bar{C}_{z j} z\right\|:=\left\|C_{z i} z\right\|, \quad j=1, \ldots, n-1 .
\end{aligned}
$$

Combined with (43), (44), and (45), (42) can be written in diagonal norm-bounded LDI form as follows [6]:

$$
\begin{gathered}
\dot{z}=A_{c l} z+B_{w} w+B_{r} r, \quad t=C_{z} z+D_{w} w, \\
\left|w_{i}\right| \leq\left\|t_{i}\right\|, \quad i=1, \ldots, n_{w} .
\end{gathered}
$$

3.3. LMI Approach for Stability Analysis. If either stabilization or regulation problem is considered, the first element $\ddot{x}_{1 d}$ of $\ddot{x}_{d}$ in (46) is zero. Furthermore, if $\ddot{x}_{d}=0$ for special cases among nonlinear systems in (13), quadratic stability of the resulting closed-loop error dynamics is defined as follows [3].

Definition 1. Let $z=0$ be an exponentially stable equilibrium point of the closed-loop error dynamics in (46) where $r=$ 0 and $A_{c l}$ is Hurwitz for the given set of controller gains; $\Theta=\left\{K_{1}, \ldots, K_{n}, \tau_{2}, \ldots, \tau_{n}\right\}$. Then, a nonlinear system in (13) is quadratically stabilizable via DSC if there exists a positive definite matrix $P$ such that

$$
\begin{aligned}
\frac{d}{d t} V(z)= & \frac{d}{d t}\left(z^{T} P z\right)=\left(A_{c l} z+B_{w} w\right)^{T} P z \\
& +z^{T} P\left(A_{c l} z+B_{w} w\right)<0 .
\end{aligned}
$$

Furthermore, the quadratic stability under the DSC is guaranteed by the following theorem.

Theorem 2. Suppose that the closed-loop error dynamics in (46) are given for the given set of controller gains,

$$
\Theta=\left\{K_{1}, \ldots, K_{n}, \tau_{2}, \ldots, \tau_{n}\right\},
$$

for all $x$ in a domain $\mathscr{D}$. If there exist $P>0$ and $\Sigma_{B}=$ $\operatorname{diag}\left(\sigma_{1}, \ldots, \sigma_{n-1}, \sigma_{n} I, \ldots, \sigma_{n_{w}} I\right) \geq 0$ such that

$$
\left[\begin{array}{cc}
A_{c l}^{T} P+P A_{c l}+C_{z}^{T} \Sigma_{B} C_{z} & P B_{w}+C_{z}^{T} \Sigma_{B} D_{w} \\
B_{w}^{T} P+D_{w}^{T} \Sigma_{B} C_{z} & D_{w}^{T} \Sigma_{B} D_{w}-\Sigma_{B}
\end{array}\right]<0,
$$

where $C_{z}=\left[\begin{array}{lll}C_{z 1}^{T} & \cdots & C_{z n_{w}}^{T}\end{array}\right]^{T}$ and $D_{w}=\left[\begin{array}{lll}D_{w 1}^{T} & \cdots & D_{z n_{w}}^{T}\end{array}\right]^{T}$, the origin in (46) is then exponentially stable in $\mathscr{D}$. Thus the nonlinear system (13) is quadratically stabilizable via DSC with the given $\Theta$ on $\mathscr{D}$.

For details of the proof of the theorem, readers are referred to Boyd et al. [6].

If a tracking problem is considered $\left(\ddot{x}_{1 d} \neq 0\right)$ and it is assumed that $\ddot{x}_{d}$ is bounded, the ultimate and quadratic boundedness is defined as follows [3].
Definition 3. The error dynamics in (46) is quadratically bounded with Lyapunov matrix $P$ if there exists $P>0$ such that

$$
\begin{array}{r}
z^{T} P z>1 \quad \text { implies }\left(A_{c l} z+B_{w} w+B_{r} r\right)^{T} P z \\
+z^{T} P\left(A_{c l} z+B_{w} w+B_{r} r\right)<0
\end{array}
$$

for all nonzero $z \in \mathscr{E}_{P}=\left\{z \in \Re^{n_{z}} \mid z^{T} P z \geq 1\right\}$.

It is noted that the assumption that $\ddot{x}_{d}$ is bounded is feasible because the time derivative of the filtered signal $x_{d}$ is bounded.

Suppose $r$ in (46) is norm-bounded such that $\|r\| \leq r_{0}$. After defining $\widetilde{r}:=r / r_{0}$ and $\widetilde{B}_{r}:=r_{0} B_{r}$, the error dynamics in (46) is written as

$$
\begin{gathered}
\dot{z}=A_{c l} z+B_{w} w+\widetilde{B}_{r} \tilde{r}, \quad t=C_{z} z+D_{w} w, \\
\left|w_{i}\right| \leq\left\|t_{i}\right\|, \quad\|\widetilde{r}\| \leq 1 .
\end{gathered}
$$

Without loss of generality, it can be considered that $\widetilde{r}$ is a unit-peak input. Then, the following theorem describes the condition for guaranteeing quadratic tracking as well as the computation of the matrix $P$ for a given set of controller gains.

Theorem 4. For the given set of controller gains, $\Theta$, suppose that the closed-loop error dynamics in (51) is given on the domain $\mathscr{D}$ and $x_{1 d}$ is a feasible output trajectory. The closed-loop error dynamics is quadratically bounded with Lyapunov matrix $P$ if there exist $P>0, \Sigma_{B}=$ $\operatorname{diag}\left(\sigma_{1}, \ldots, \sigma_{n-1}, \sigma_{n} I, \ldots, \sigma_{n_{w}} I\right) \geq 0$, and $\alpha \geq 0$ such that

$$
\left[\begin{array}{ccc}
A_{c l}^{T} P+P A_{c l}+\alpha P+C_{z}^{T} \Sigma_{B} C_{z} & P B_{w}+C_{z}^{T} \Sigma_{B} D_{w} & P \widetilde{B}_{r} \\
B_{w}^{T} P+D_{w}^{T} \Sigma_{B} C_{z} & D_{w}^{T} \Sigma_{B} D_{w}-\Sigma_{B} & 0 \\
\widetilde{B}_{r}^{T} P & 0 & -\alpha I
\end{array}\right]<0,
$$

where $\widetilde{B}_{r}=r_{0} B_{r}, C_{z}=\left[\begin{array}{lll}C_{z 1}^{T} & \cdots & C_{z n_{w}}^{T}\end{array}\right]^{T}$, and $D_{w}=$ $\left[\begin{array}{lll}D_{w 1}^{T} & \cdots & D_{z n_{w}}^{T}\end{array}\right]^{T}$.

Readers are referred to Boyd et al. [6] for the proof and the definition of the feasible output trajectory is explained in $[3,4]$.

3.4. Illustrative Example. Consider the example in (2) and (3) where $f_{1}=-x_{1}^{2}$ and the domain $\mathscr{D}$ is defined as

$$
\mathscr{D}=\left\{x \in \mathfrak{R}^{2}|| x_{1}|\leq 1,| x_{2} \mid \leq \frac{\pi}{4}\right\} .
$$

Then, (5) becomes

$$
\bar{g}_{2}\left(\bar{x}_{2}\right)=\tan \bar{x}_{2}=x_{1}^{2}-K S_{1},
$$

and a first order low-pass filter is defined as

$$
\tau \dot{g}_{2 d}+g_{2 d}=\bar{g}_{2}, \quad g_{2 d}(0)=\bar{g}_{2}(0) .
$$

It is noted that $\bar{g}_{2}$ instead of $\bar{x}_{2}$ is passed through the low-pass filter. After defining the second sliding surface $S_{2}=x_{2}-x_{2 d}$, 
where $x_{2 d}=\tan ^{-1}\left(g_{2 d}\right)$, and taking its derivative, the control input is obtained by

$$
u=\dot{x}_{2 d}-K S_{2}
$$

where

$$
\dot{x}_{2 d}=\frac{d}{d t} \tan ^{-1}\left(g_{2 d}\right)=\frac{1}{1+g_{2 d}^{2}} \dot{g}_{2 d}=\frac{1}{1+g_{2 d}^{2}} \frac{\bar{g}_{2}-g_{2 d}}{\tau}
$$

and the last equality comes from (55).

If a new filter error, $\xi_{2}=g_{2 d}-\bar{g}_{2}$, is defined, the augmented closed-loop dynamics can be written as

$$
\begin{aligned}
\dot{S}_{1} & =-K S_{1}+\xi_{2}+\left(\tan x_{2}-\tan x_{2 d}\right)=-K S_{1}+\xi_{2}+h_{2}, \\
\dot{S}_{2} & =-K S_{2}, \\
\dot{\xi}_{2} & =\frac{d}{d t}\left(g_{2 d}-\bar{g}_{2}\right)=-\frac{\xi_{2}}{\tau}+\frac{d}{d t}\left\{f_{1}+K S_{1}\right\} \\
& =-\frac{\xi_{2}}{\tau}+\dot{f}_{1}+K \dot{S}_{1},
\end{aligned}
$$

where $h_{2}=\tan x_{2}-\tan x_{2 d}$ and $\dot{g}_{2 d}=-\xi_{2} / \tau$ from (55). Therefore, (58) is written in matrix form as

$$
\begin{gathered}
{\left[\begin{array}{ccc}
1 & 0 & 0 \\
0 & 1 & 0 \\
-K & 0 & 1
\end{array}\right]\left[\begin{array}{l}
\dot{S}_{1} \\
\dot{S}_{2} \\
\dot{\xi}_{2}
\end{array}\right]} \\
=\left[\begin{array}{ccc}
-K & 0 & 1 \\
0 & -K & 0 \\
0 & 0 & -\frac{1}{\tau}
\end{array}\right]\left[\begin{array}{l}
S_{1} \\
S_{2} \\
\xi_{2}
\end{array}\right]+\left[\begin{array}{ll}
1 & 0 \\
0 & 0 \\
0 & 1
\end{array}\right]\left[\begin{array}{c}
h_{2} \\
\dot{f}_{1}
\end{array}\right], \\
{\left[\begin{array}{c}
\dot{S}_{1} \\
\dot{S}_{2} \\
\dot{\xi}_{2}
\end{array}\right]=\left[\begin{array}{ccc}
-K & 0 & 1 \\
0 & -K & 0 \\
-K^{2} & 0 & K-\frac{1}{\tau}
\end{array}\right]\left[\begin{array}{l}
S_{1} \\
S_{2} \\
\xi_{2}
\end{array}\right]+\left[\begin{array}{ll}
1 & 0 \\
0 & 0 \\
K & 1
\end{array}\right]\left[\begin{array}{l}
h_{2} \\
\dot{f}_{1}
\end{array}\right] .}
\end{gathered}
$$

The upper bound of $\left[\begin{array}{ll}h_{2} & \dot{f}_{1}\end{array}\right]^{T}$ can be determined as

$$
\begin{aligned}
\left|h_{2}\right| & =\left|\tan x_{2}-\tan x_{2 d}\right| \leq\left|x_{2}-x_{2 d}\right| \\
& =\left|S_{2}\right|=\left|\left[\begin{array}{lll}
0 & 1 & 0
\end{array}\right] z\right|:=\left|c_{z 1} z\right|, \\
\left|\dot{f}_{1}\right| & =\left|\frac{\partial f_{1}}{\partial x_{1}} \dot{x}_{1}\right|=\left|-2 x_{1} \dot{x}_{1}\right|=\left|-2 x_{1}\left(-K S_{1}+\xi_{2}+h_{2}\right)\right| \\
& \leq 2\left|-K S_{1}+\xi_{2}+h_{2}\right|=2\left|\left[\begin{array}{lll}
-K & 0 & 1
\end{array}\right] z+\left[\begin{array}{ll}
1 & 0
\end{array}\right] w\right| \\
& :=\left|c_{z 2} z+d_{w 2} w\right|,
\end{aligned}
$$

where the first inequality comes from a Lipschitz condition such that $|\tan x-\tan y| \leq|x-y|$ for all $x, y \in \mathscr{D}$ and the second inequality comes from a fact that $\partial f_{1} / \partial x_{1}$ is bounded on $\mathscr{D}$.

Finally, the augmented error dynamics can be written in diagonal norm-bounded LDI form as follows:

$$
\begin{aligned}
\dot{z}=A_{c l} z+B_{w} w, & t=C_{z} z+D_{w} w \\
\left|w_{i}\right| \leq\left|t_{i}\right|, & i=1,2
\end{aligned}
$$
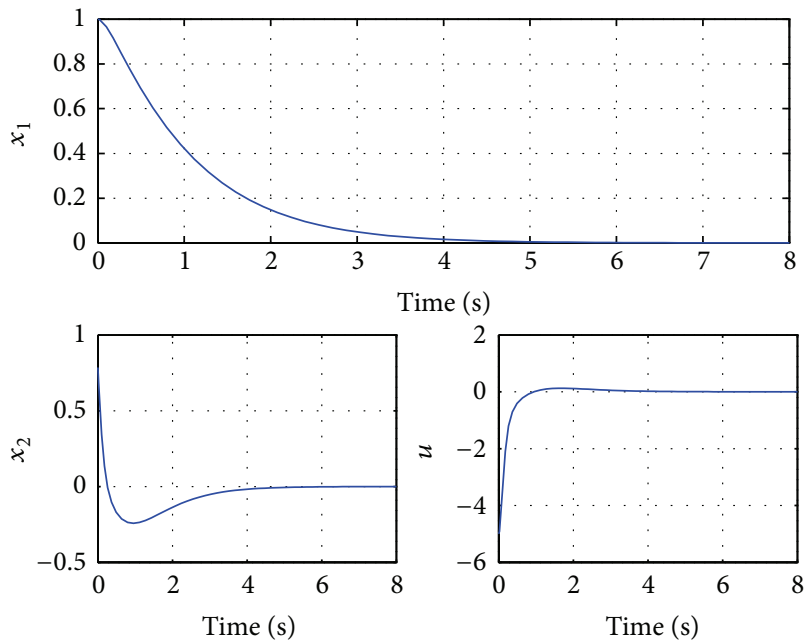

FIGURE 1: Time responses of $x$ and $u$ for the given initial condition, $\left[x_{1}(0) x_{2}(0)\right]=\left[\begin{array}{ll}1 & \pi / 4\end{array}\right]$.

where

$$
C_{z}=\left[\begin{array}{c}
c_{z 1} \\
c_{z 2}
\end{array}\right]=\left[\begin{array}{ccc}
0 & 1 & 0 \\
-2 K & 0 & 2
\end{array}\right], \quad D_{w}=\left[\begin{array}{c}
0_{2}^{T} \\
d_{w 2}
\end{array}\right]=\left[\begin{array}{ll}
0 & 0 \\
2 & 0
\end{array}\right] .
$$

When the controller gains are given as $K=1$ and $\tau=0.1$, LMI (49) is solved numerically in the framework of convex optimization using CVX [8]. It is shown that the closedloop system is quadratically stable by finding the feasible solution of LMI (49). For the given controller gains and initial condition, the time responses of $x$ and $u$ are shown in Figure 1 and $x(t) \rightarrow 0$ as $t \rightarrow \infty$. Thus, it is validated that the result of quadratic stability analysis based on an LMI approach is equivalent to simulation results.

\section{Application to Lateral Vehicle Control}

The proposed control approach is applied to design the robust lateral control algorithm for autonomous valet parking (AVP). It is assumed that the position and heading angle information is provided via either infrastructure sensors and vehicle to infrastructure (V2I) communication [9] or an invehicle sensor such as DGPS [10]. The objective of the lateral controller is to perform two different maneuvers for AVP, that is, forward driving and backward parallel parking. Therefore, it is necessary for the lateral controller to be robust enough to track desired trajectories for different driving maneuvers.

4.1. Vehicle Model. While the bicycle model has been used widely for design of a lateral controller for high speed driving on highway [11, 12], a vehicle is driving at low speed for AVP and thus slip angle can be neglected in this study. Therefore, 


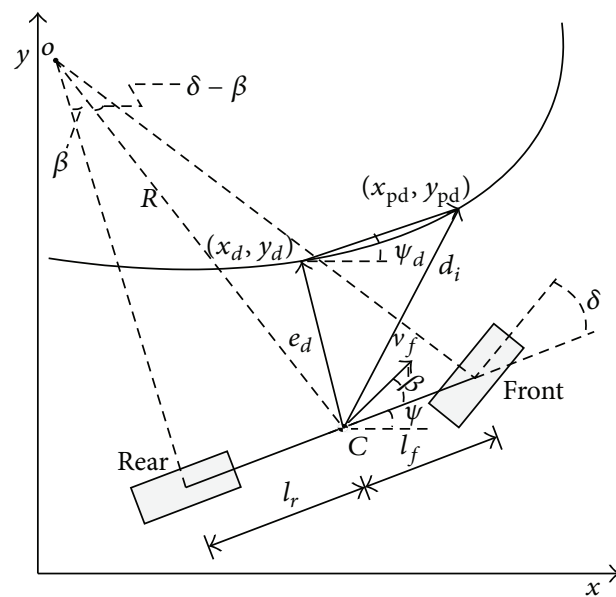

FIGURE 2: Schematic of kinematic model and error definition.

the following kinematic model is used for both forward driving and backward parallel parking (refer to Figure 2) [13]:

$$
\begin{aligned}
\dot{x} & =v_{i} \cos (\psi+\beta), \\
\dot{y} & =v_{i} \sin (\psi+\beta), \\
\dot{\psi} & =\frac{v_{i}}{l} \cos \beta \tan \delta=\frac{v_{i}}{l_{j}} \sin \beta:=\frac{v_{i}}{l_{j}} g_{2}(\beta),
\end{aligned}
$$

where the subscript $i$ represents the driving maneuver; that is, $i=f$ for forward driving and $i=r$ for backward parking,

$$
\begin{gathered}
\beta=\tan ^{-1}\left(\frac{l_{i}}{l} \tan \delta\right), \quad v_{i}= \begin{cases}v_{f} & \text { for } i=f, \\
-v_{r} & \text { for } i=r,\end{cases} \\
j= \begin{cases}r & \text { for } i=f, \\
f & \text { for } i=r .\end{cases}
\end{gathered}
$$

If dynamics of a steering actuator from steering wheel angle command to steering angle of the vehicle is considered, the following equation of motion may be added:

$$
\tau_{s} \dot{\theta}+\theta=u, \quad|\delta|=\left|R_{s} \theta\right| \leq \delta_{\max },
$$

where $\delta_{\max }$ is the maximum steering angle.

4.2. Controller Design. With consideration of operating conditions such as low speed and small slip angle, the nonlinear kinematic model with actuator dynamics in (63) and (65) is used for design of the lateral controller. Then, the proposed control approach based on DSC is applied to the kinematic model as follows. First, the first error surface is defined using the idea of preview control suggested in [14] (see in Figure 2):

$$
S_{1 i}=e_{b i}+d_{i} e_{\psi}=e_{b}+d_{i}\left(\psi_{d}-\psi\right),
$$

where the lateral error $e_{b}$ is defined as

$$
\begin{aligned}
e_{b i} & = \begin{cases}0 & \text { if } c=0, \\
-\operatorname{sign}(c) \cdot\left\|e_{d}\right\| & \text { otherwise, }\end{cases} \\
e_{d} & =\left[\begin{array}{l}
x_{d}-x \\
y_{d}-y
\end{array}\right]:=\left[\begin{array}{l}
e_{x} \\
e_{y}
\end{array}\right], \\
a & =\left[\begin{array}{l}
x_{p d}-x_{d} \\
y_{p d}-y_{d}
\end{array}\right] \in \mathfrak{R}^{2}, \quad b=\left[\begin{array}{l}
x-x_{d} \\
y-y_{d}
\end{array}\right] \in \mathfrak{R}^{2}, \\
c & =\operatorname{det}\left(\left[\begin{array}{l}
a^{T} \\
b^{T}
\end{array}\right]\right),
\end{aligned}
$$

the point $\left(x_{d}, y_{d}\right)$ is the closest point with respect to current position, and the preview distance $d_{i}$ and desired heading angle $\psi_{d}$ are

$$
d_{i}=\left\|\left[\begin{array}{l}
x_{p d}-x \\
y_{p d}-y
\end{array}\right]\right\|, \quad \psi_{d}=\tan ^{-1}\left(\frac{x_{p d}-x_{d}}{y_{p d}-y_{d}}\right) .
$$

For instance, $\operatorname{sign}(c)$ is negative for the given scenario in Figure 2 because the rotational direction from vector $a$ to $b$ defined in (67) is clockwise. Thus, the resulting positive lateral error implies a steering wheel angle command in the counterclockwise direction. If it is assumed that the preview distance is a constant, the point $\left(x_{p d}, y_{p d}\right)$ in Figure 2 can be calculated with respect to the given desired trajectory and the desired heading angle is then determined.

After taking a derivative of $S_{1 i}$ along the trajectory of (63), the derivative of $S_{1 i}$ is

$$
\dot{S}_{1 i}=\dot{e}_{b i}+d_{i} \dot{\psi}_{d}-\frac{d_{i} v_{i}}{l_{j}} \sin \beta .
$$

It is remarked that $d_{i}$ can be chosen as a variable if necessary. For the simplicity of derivation, it is assumed to be constant. To make $S_{1 i}$ go to zero, let $\dot{S}_{1 i}=-K_{1 i} S_{1 i}$, where $K_{1 i}$ is a controller gain. Then the desired steering angle is obtained as

$$
\bar{g}_{2}(\bar{\beta})=\sin \bar{\beta}=\frac{l_{j}}{d_{i} v_{i}}\left(\dot{e}_{b i}+d_{i} \dot{\psi}_{d}+K_{1 i} S_{1 i}\right),
$$

where

$$
\begin{aligned}
\dot{e}_{b i}= & \operatorname{sign}(c) \cdot \dot{e}_{d}, \\
\dot{e}_{d}= & \frac{e_{x}+e_{y}}{e_{d}}\left(\dot{e}_{x}+\dot{e}_{y}\right) \\
= & \frac{e_{x}+e_{y}}{e_{d}}\left\{\left(\dot{x}_{d}-v_{i} \cos (\psi+\beta)\right)\right. \\
& \left.\quad+\left(\dot{y}_{d}-v_{i} \sin (\psi+\beta)\right)\right\},
\end{aligned}
$$




$$
\begin{aligned}
\dot{\psi}_{d}= & \frac{d}{d t} \tan ^{-1}\left(\frac{x_{p d}-x_{d}}{y_{p d}-y_{d}}\right) \\
= & \frac{1}{1+\left(\left(x_{p d}-x_{d}\right) /\left(y_{p d}-y_{d}\right)\right)^{2}} \\
& \times \frac{\left(\dot{x}_{p d}-\dot{x}_{d}\right)\left(y_{p d}-y_{d}\right)-\left(x_{p d}-x_{d}\right)\left(\dot{y}_{p d}-\dot{y}_{d}\right)}{\left(y_{p d}-y_{d}\right)^{2}} .
\end{aligned}
$$

It is noted that all of $\dot{x}_{d}, \dot{x}_{p d}, \dot{y}_{d}$, and $\dot{y}_{p d}$ are known for the given desired trajectory.

Then, the second error surface is defined as $S_{2 i}=\beta-\beta_{\text {des }}$, where $\beta_{\text {des }}=q_{2}\left(g_{2 d}\right)=\sin ^{-1}\left(g_{2 d}\right)$ and $g_{2 d}$ is calculated after passing through a first order low-pass filter as follows:

$$
\tau_{2} \dot{g}_{2 d}+g_{2 d}=\bar{g}_{2} .
$$

After differentiating $S_{2 i}$ and using (65), the resulting equation is

$$
\begin{aligned}
\dot{S}_{2 i} & =\dot{\beta}-\dot{\beta}_{\mathrm{des}} \\
& =\frac{l_{j}\left(1+\tan ^{2} \delta\right)}{l\left(1+\left(l_{r}^{2} / l^{2}\right) \tan ^{2} \delta\right)} R_{s} \dot{\theta}-\dot{\beta}_{\mathrm{des}} \\
& =\frac{R_{s} l_{j}\left(1+\tan ^{2} \delta\right)}{l\left(1+\left(l_{j}^{2} / l^{2}\right) \tan ^{2} \delta\right)} \frac{u-\theta}{\tau_{s}}-\dot{\beta}_{\mathrm{des}},
\end{aligned}
$$

where

$$
\dot{\beta}=\frac{d}{d t} \tan ^{-1}\left(\frac{l_{j} \tan \delta}{l}\right)=\frac{1}{1+\left(l_{j} \tan \delta / l\right)^{2}} \frac{l_{j}}{l} \sec ^{2} \delta \cdot \dot{\delta} .
$$

Let $\dot{S}_{2 i}=-K_{2 i} S_{2 i}$, where $K_{2 i}$ is a controller gain. Then the desired steering wheel angle is obtained as

$$
u=\theta+\frac{\tau_{s}\left(l^{2}+l_{j}^{2} \tan ^{2} \delta\right)}{R_{s} \cdot l \cdot l_{j}\left(1+\tan ^{2} \delta\right)}\left(\dot{\beta}_{\mathrm{des}}-K_{2 i} S_{2 i}\right),
$$

where

$$
\dot{\beta}_{\mathrm{des}}=\frac{\partial q_{2}}{\partial g_{2 d}} \dot{g}_{2 d}=\frac{1}{\sqrt{1-g_{2 d}^{2}}} \frac{\bar{g}_{2}-g_{2 d}}{\tau_{2}},
$$

and the last equality comes from (72).

4.3. Stability Analysis. If $\sin \beta_{\mathrm{des}}$ and $\sin \bar{\beta}$ are added and subtracted in (69) and $u$ in (75) is put in (73), the augmented closed-loop error dynamics is

$$
\begin{aligned}
\dot{S}_{1 i}= & \dot{e}_{b i}+d_{i} \dot{\psi}_{d}-\frac{d_{i} v_{i}}{l_{j}}\left(\sin \beta-\sin \beta_{\mathrm{des}}\right) \\
& -\frac{d_{i} v_{i}}{l_{j}}\left(\sin \beta_{\mathrm{des}}-\sin \bar{\beta}\right)-\frac{d_{i} v_{i}}{l_{j}} \sin \bar{\beta},
\end{aligned}
$$

$$
\begin{aligned}
& \dot{S}_{2 i}=-K_{2 i} S_{2 i}, \\
& \dot{\xi}_{2 i}=\dot{g}_{2 d}-\dot{\bar{g}}_{2}=-\frac{\xi_{2}}{\tau_{2}}-\frac{l_{j}}{d_{i} v_{i}}\left(\ddot{e}_{y i}+d_{i} \ddot{\psi}_{d}+K_{1 i} \dot{S}_{1 i}\right),
\end{aligned}
$$

where it is assumed that $v_{i}$ is constant with respect to time. Using (70), (77) is written as

$$
\begin{aligned}
\dot{S}_{1 i} & =-K_{1 i} S_{1 i}-\frac{d_{i} v_{i}}{l_{j}} \xi_{2 i}-\frac{d_{i} v_{i}}{l_{j}} h_{2}, \\
\dot{S}_{2 i} & =-K_{2 i} S_{2 i}, \\
\frac{l_{j}}{d_{i} v_{i}} K_{1 i} \dot{S}_{1 i}+\dot{\xi}_{2 i} & =-\frac{\xi_{2}}{\tau_{2}}-\frac{l_{j}}{d_{i} v_{i}}\left(\ddot{e}_{b i}+d_{i} \ddot{\psi}_{d}\right),
\end{aligned}
$$

where $h_{2}=\sin \beta-\sin \beta_{\text {des }}$. As done in the example of Section 3.4, the augmented error dynamics is written in matrix form as follows:

$$
\begin{aligned}
& {\left[\begin{array}{ccc}
1 & 0 & 0 \\
0 & 1 & 0 \\
\frac{l_{j} K_{1 i}}{\left(d_{i} v_{i}\right)} & 0 & 1
\end{array}\right]\left[\begin{array}{l}
\dot{S}_{1 i} \\
\dot{S}_{2 i} \\
\dot{\xi}_{2 i}
\end{array}\right]} \\
& =\left[\begin{array}{ccc}
-K_{1 i} & 0 & -\frac{d_{i} v_{i}}{l_{j}} \\
0 & -K_{2 i} & 0 \\
0 & 0 & -\frac{1}{\tau_{2}}
\end{array}\right]\left[\begin{array}{l}
S_{1 i} \\
S_{2 i} \\
\xi_{2 i}
\end{array}\right] \\
& +\left[\begin{array}{c}
-\frac{d_{i} v_{i}}{l_{j}} \\
0 \\
0
\end{array}\right] h_{2}+\left[\begin{array}{cc}
0 & 0 \\
0 & 0 \\
-\frac{l_{j}}{\left(d_{i} v_{i}\right)} & -\frac{l_{j}}{v_{i}}
\end{array}\right]\left[\begin{array}{l}
\ddot{e}_{b i} \\
\ddot{\psi}_{d}
\end{array}\right] \text {, } \\
& {\left[\begin{array}{l}
\dot{S}_{1 i} \\
\dot{S}_{2 i} \\
\dot{\xi}_{2 i}
\end{array}\right]=\left[\begin{array}{ccc}
-K_{1 i} & 0 & -\frac{d_{i} v_{i}}{l_{j}} \\
0 & -K_{2 i} & 0 \\
\frac{l_{j} K_{1 i}^{2}}{\left(d_{i} v_{i}\right)} & 0 & K_{1 i}-\frac{1}{\tau_{2}}
\end{array}\right]\left[\begin{array}{l}
S_{1 i} \\
S_{2 i} \\
\xi_{2 i}
\end{array}\right]} \\
& +\left[\begin{array}{c}
-\frac{d_{i} v_{i}}{l_{j}} \\
0 \\
K_{1 i}
\end{array}\right] h_{2}-\frac{l_{j}}{v_{i}}\left[\begin{array}{cc}
0 & 0 \\
0 & 0 \\
\frac{1}{d_{i}} & 1
\end{array}\right]\left[\begin{array}{c}
\ddot{e}_{b i} \\
\ddot{\psi}_{d}
\end{array}\right] .
\end{aligned}
$$

Furthermore, the upper bound of $h_{2}$ is obtained as $\left|h_{2}\right|=$ $\left|\sin \beta-\sin \beta_{\text {des }}\right| \leq\left|\beta-\beta_{\text {des }}\right|=\left|S_{2}\right|$ because it is Lipschitz, and both $\dot{e}_{b i}$ and $\dot{\psi}_{d}$ in (71) are differentiable on $\mathscr{D} ;\left[\begin{array}{ll}\ddot{e}_{b i} & \ddot{\psi}_{d}\end{array}\right]^{T}$ is bounded with respect to the desired trajectory. Thus, without loss of generality, it is assumed that $\left[\begin{array}{ll}\ddot{e}_{b i} & \ddot{\psi}_{d}\end{array}\right]^{T}$ is norm-bounded such that $\left\|\left[\begin{array}{ll}\ddot{e}_{b i} & \ddot{\psi}_{d}\end{array}\right]^{T}\right\| \leq r_{0}$. Therefore, the 

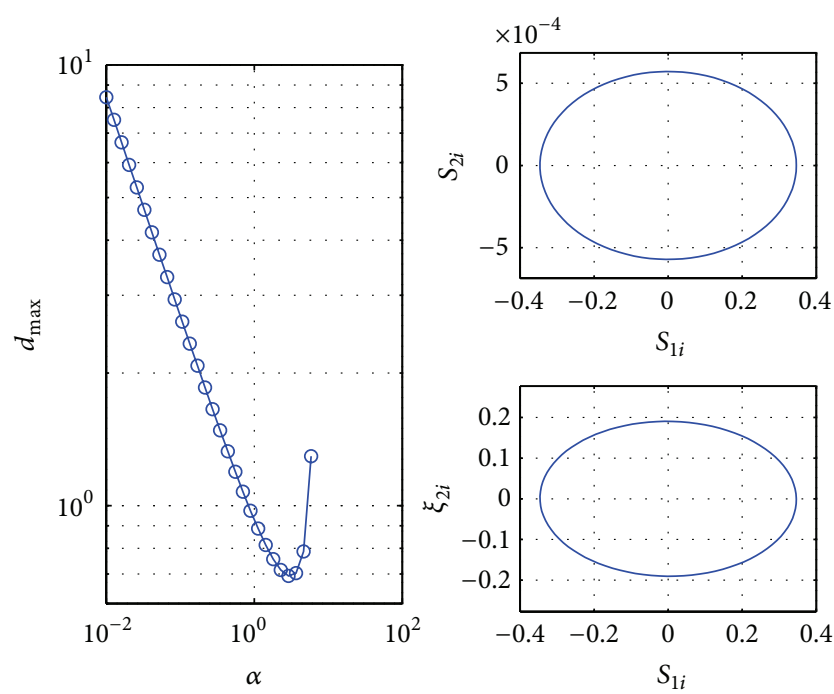

FIGURE 3: Minimum of the maximum diameter of ellipsoid along line search of $\alpha$.

augmented error dynamics can be written in LDI form of (51) as

$$
\begin{gathered}
\dot{z}_{i}=A_{i} z_{i}+B_{w} w+\widetilde{B}_{r} \widetilde{r}_{i}, \quad t=C_{z} z, \quad i=f, r, \\
|w| \leq|t|, \quad\|\widetilde{r}\| \leq 1,
\end{gathered}
$$

where $z_{i}=\left[\begin{array}{lll}S_{1 i} & S_{2 i} & \xi_{2 i}\end{array}\right]^{T} \in \mathfrak{R}^{3}, w=h_{2}$, and $C_{z}=\left[\begin{array}{lll}0 & 1 & 0\end{array}\right]$.

Suppose $v_{i}=3(\mathrm{~m} / \mathrm{s}), l_{j}=1.35(\mathrm{~m})$ in $(79)$, and the control parameters are assigned as $K_{1 i}=K_{2 i}=3, \tau_{2}=0.02$, and $d_{f}=10$ and $d_{r}=1$. When $r_{0}$ is assumed to be 5, LMI (52) is solved iteratively for a fixed $\alpha$ by minimizing the largest semiaxis (i.e., maximizing the smallest eigenvalue of $P$ ) [3]. That is, after the 40 logarithmically equally spaced points between $10^{-2}$ and $10^{2}$ are generated for $\alpha$ 's, the minimum of the maximum diameter, which is $d_{\max }=2 / \sqrt{\lambda_{\min }(P)}$, is obtained when $\alpha=2.8943$ (in the left plot of Figure 3). Then the 20 linearly equally spaced points between 0.3455 and 0.5541 are generated and the iterative computation of LMI (52) is performed for each $\alpha$. Finally, for $\alpha=0.5212$, the corresponding maximum diameter of the ellipsoid, $d_{\max }$, is 0.6925 which is the semiaxis in the $S_{2 i}$ axis. It is remarked that the size of the ultimate and quadratic error bound is roughly proportional to the magnitude of $r_{0}$ and thus more accurate estimation of the error bound relies on better estimate of $r_{0}$ resulting from the desired trajectory. In consequence, it is expected that $S_{1}$ is bounded for the given set of control parameters. Furthermore, the relative degree of the error dynamics is one and it is shown that its internal dynamics is input-state stable [14]. Thus, this implies that both $e_{b i}$ and $e_{\psi}$ are bounded if $S_{1}$ is bounded.

4.4. Simulation Results. Suppose 24 waypoints are given a priori as shown in Figure 4. Forward driving maneuver is assigned from the first waypoint to $23 \mathrm{rd}$ waypoint and backward parallel parking maneuver is requested from 23rd to 24th waypoint. Moreover, both straight and curved road

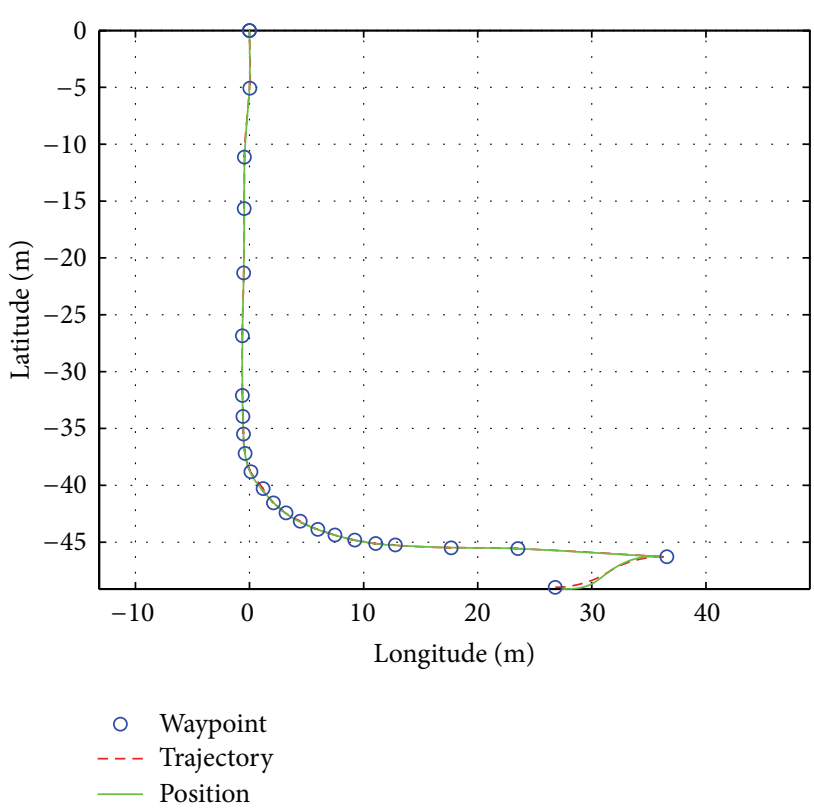

Figure 4: Desired trajectory and position of a vehicle for autonomous valet parking.

geometry is considered in this driving scenario; that is, from the first to 7 th waypoints are for the straight road, from 7 th to 20 th waypoint for the curved road with about $12(\mathrm{~m})$ radius of curvature, and from 20th to 23rd waypoint again for the straight road as shown in Figure 4.

Let the additional system parameters in (63) and (65) be $\tau_{s}=0.1, \delta_{\max }=36$ (degree), and $R_{s}=1 / 17.12$ for simulations. When the control parameters used for stability analysis above are applied to the proposed lateral controller, time responses of steering and heading angle are shown in Figure 5 and it is shown that the corresponding lateral error, $e_{b}$, is less than $0.3(\mathrm{~m})$. That is, it is shown that the lateral error is bounded as expected above. It is validated that the proposed controller enables the vehicle to perform two different maneuvers with only different value of $d_{i}$.

\section{Conclusions}

This paper developed the analysis and design method of DSC for a class of nonlinear systems where the nonlinear functions are included as forcing terms of DSC. The proposed control approach was applied to lateral vehicle control for forward driving and parallel parking maneuvers at low speed. With modification of input variables to the filter dynamics, it was shown that most of results in [3] could be used for the new class of nonlinear systems. Thus, the stability conditions in linear matrix inequality form were presented to guarantee the quadratic stability and boundedness via DSC for the given class of nonlinear systems. Furthermore, the quadratic Lyapunov functions were calculated numerically in the framework of convex optimization for a lateral vehicle control problem as well as an illustrative example. It was validated that the analysis results agreed with ones of simulation. 

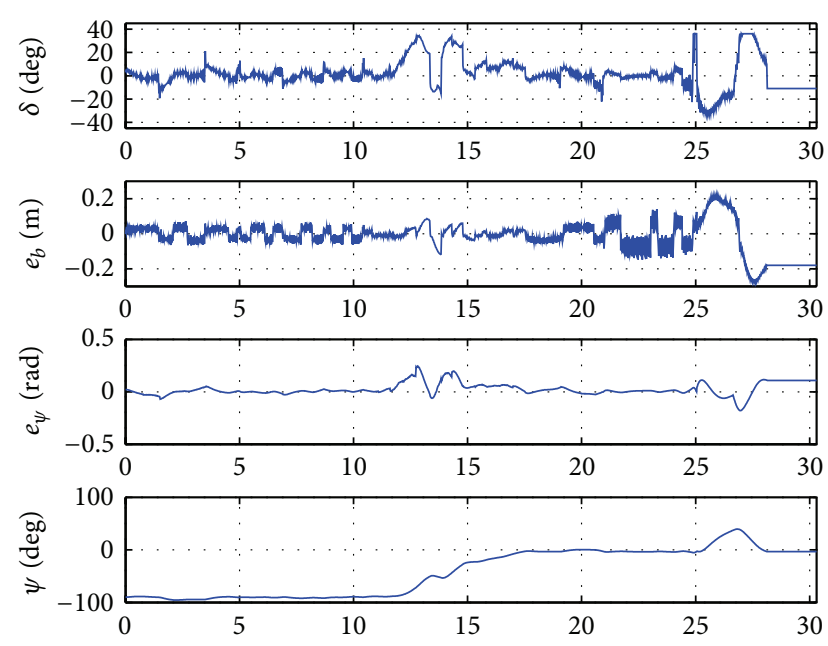

FIGURE 5: Time responses of errors, steering, and heading angle.

\section{Nomenclature}

$x$ : Vehicle position in longitudinal direction

$y$ : Vehicle position in lateral direction

$v$ : Vehicle velocity

$l_{f}$ : Length from a center of mass to front wheel axle

$l_{r}$ : Length from a center of mass to rear wheel axle

$l$ : Length of wheelbase, that is, $l=l_{f}+l_{r}$

$\delta$ : Front steering angle

$\theta$ : Steering wheel angle

$u$ : Steering wheel angle command

$R_{s}$ : Steering gear ratio, that is, $R_{s}=\delta / \theta$

$e_{b}$ : Lateral position error

$\psi$ : Heading angle

$e_{\psi}$ : Heading angle error

$d_{i}$ : Preview distance

$\tau_{s}$ : Time constant for steering actuator

$\beta$ : Slip angle.

\section{Conflict of Interests}

The authors declare that there is no conflict of interests regarding the publication of this paper.

\section{Acknowledgments}

This work was supported in part by Basic Science Research Program through the National Research Foundation of Korea (NRF) funded by the Ministry of Education, Science and Technology (no. 2009-0075110). It was also supported in part by the research project funded by LS Mtron.

\section{References}

[1] J. K. Hedrick and P. P. Yip, "Multiple sliding surface control: Theory and application," Journal of Dynamic Systems, Measurement and Control, vol. 122, no. 4, pp. 586-593, 2000.

[2] D. Chwa, "Global tracking control of underactuated ships with input and velocity constraints using dynamic surface control method," IEEE Transactions on Control Systems Technology, vol. 19, no. 6, pp. 1357-1370, 2011.

[3] B. Song and J. K. Hedrick, Dynamic Surface Control of Uncertain Nonlinear Systems: An LMI Approach, Springer, New York, NY, USA, 2011.

[4] D. Swaroop, J. K. Hedrick, P. P. Yip, and J. C. Gerdes, “Dynamic surface control for a class of nonlinear systems," IEEE Transactions on Automatic Control, vol. 45, no. 10, pp. 1893-1899, 2000.

[5] J. C. Gerdes and J. K. Hedrick, "Loop-at-a-time' design of dynamic surface controllers for nonlinear systems," Journal of Dynamic Systems, Measurement and Control, vol. 124, no. 1, pp. 104-110, 2002.

[6] S. Boyd, L. El Ghaoui, E. Feron, and V. Balakrishnan, Linear Matrix Inequalities in System and Control Theory, vol. 15 of SIAM Studies in Applied Mathematics, SIAM, Philadelphia, Pa, USA, 1994.

[7] H. K. Khalil, Nonlinear Systems, Prentice Hall, New York, NY, USA, 3rd edition, 2002.

[8] M. Grant and S. Boyd, "CVX: matlab software for disciplined convex programming," version 1.21, 2011, http://cvxr.com/cvx/.

[9] B. Song, "Copperative lateral control for autonomous valet parking," International Journal of Autonomotive Technology, vol. 14, no. 4, pp. 633-640, 2013.

[10] M. Omae, H. Shimizu, and T. Fujioka, "GPS-based automatic driving control in local area with course of large curvature and parking space," Vehicle System Dynamics, vol. 42, no. 1-2, pp. 5973, 2004.

[11] H. Peng and M. Tomizuka, "Preview control for vehicle lateral guidance in highway automation," Journal of Dynamic Systems, Measurement and Control, vol. 115, no. 4, pp. 679-686, 1993.

[12] R. Rajamani, H.-S. Tan, B. K. Law, and W.-B. Zhang, "Demonstration of integrated longitudinal and lateral control for the operation of automated vehicles in platoons," IEEE Transactions on Control Systems Technology, vol. 8, no. 4, pp. 695-708, 2000.

[13] R. Rajamani, Vehicle Dynamics and Control, Springer, New York, NY, USA, 2006.

[14] R. Rajamani, C. Zhu, and L. Alexander, "Lateral control of a backward driven front-steering vehicle," Control Engineering Practice, vol. 11, no. 5, pp. 531-540, 2003. 


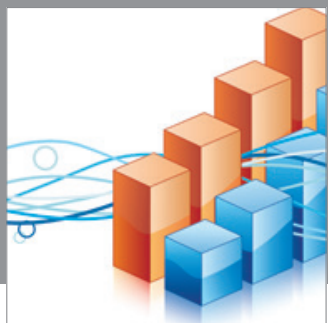

Advances in

Operations Research

mansans

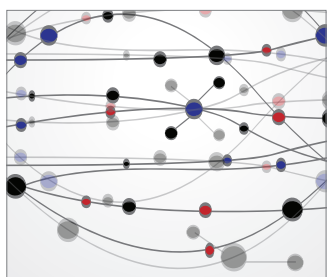

The Scientific World Journal
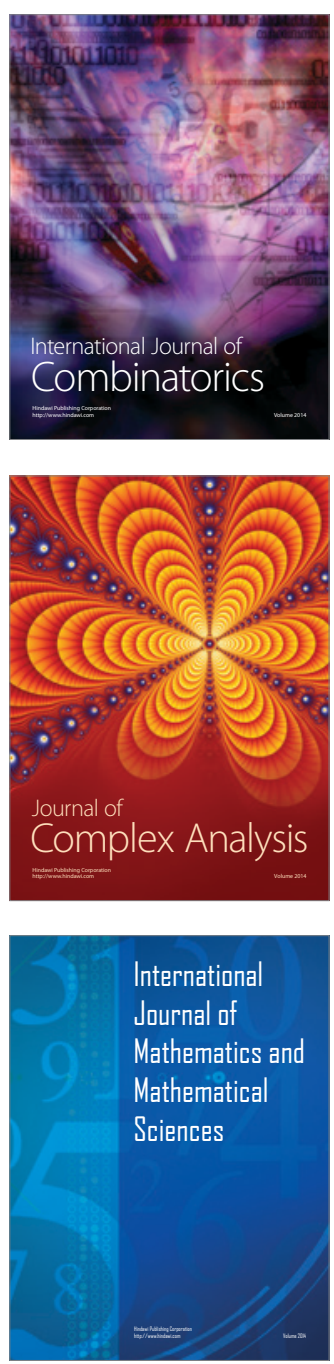
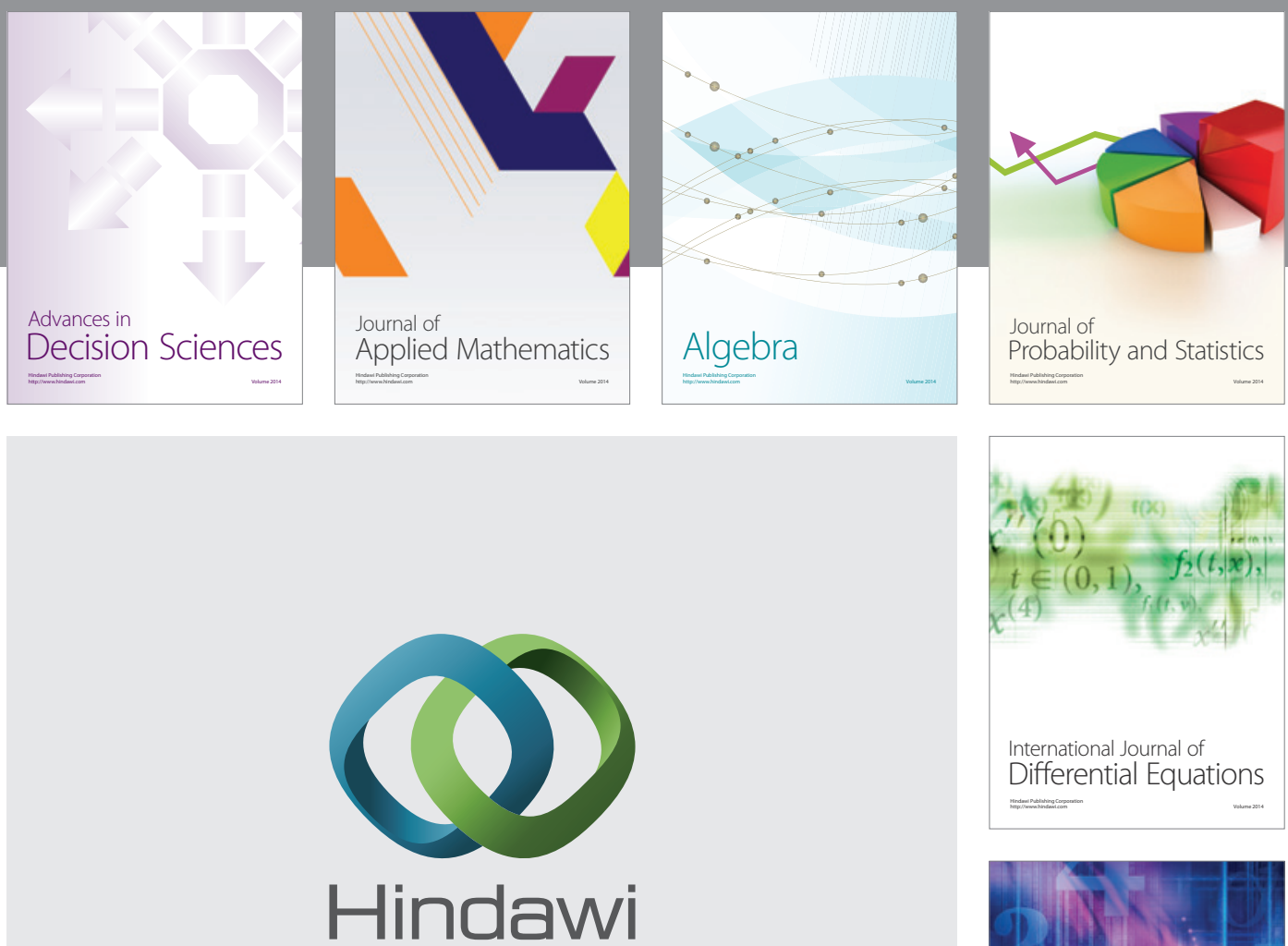

Submit your manuscripts at http://www.hindawi.com
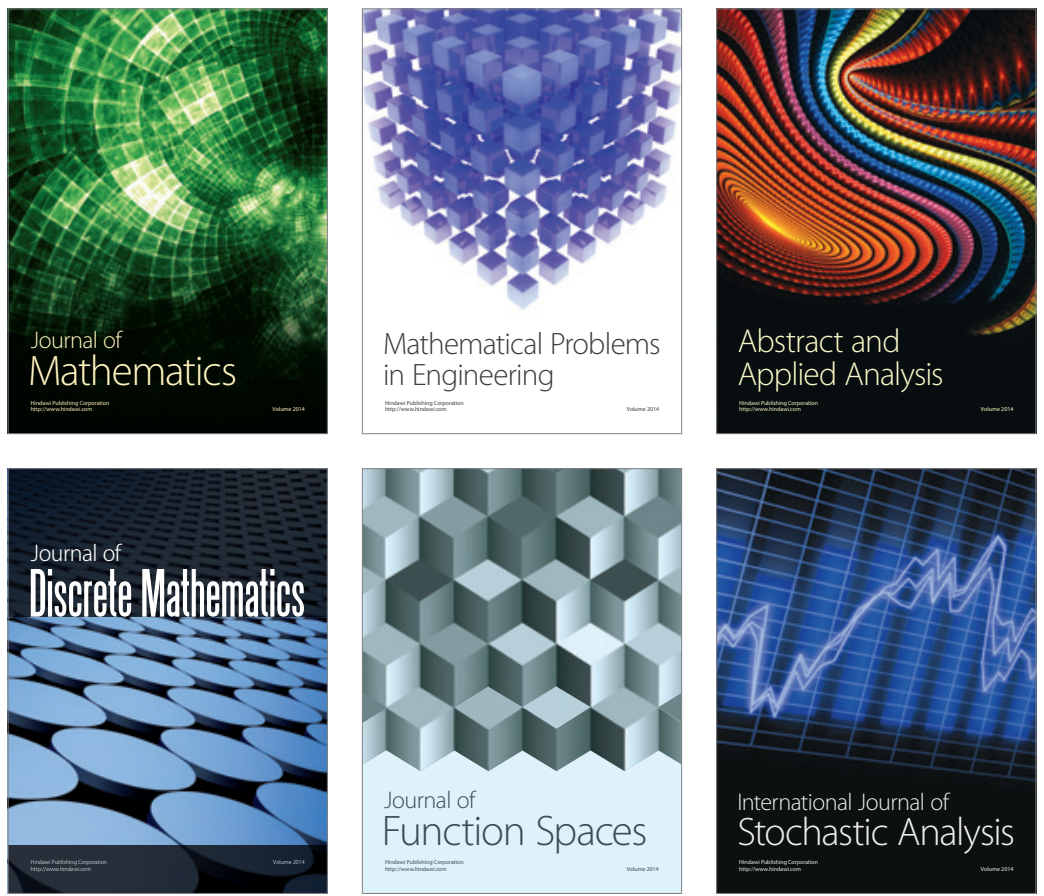

Journal of

Function Spaces

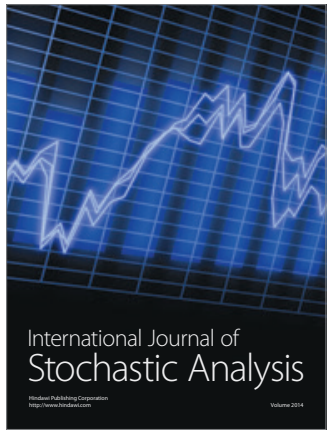

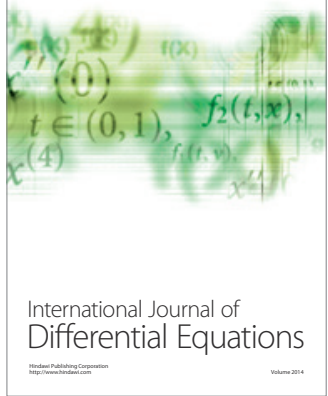
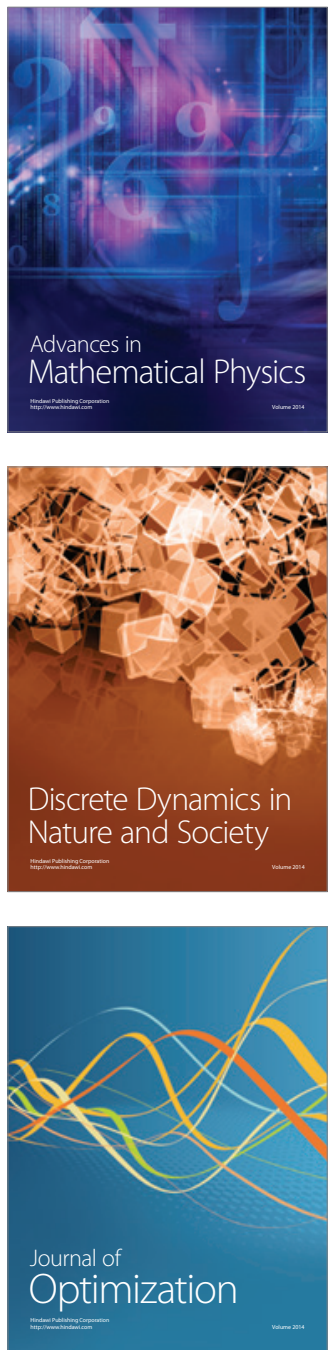